\title{
Impact of chronic kidney disease on fetomaternal outcome: a case report
}

\author{
Neha Khairnar*, Ananya Bora, Amarjeet Kaur Bava
}

Department of Obstetrics and Gynecology, LTMMC and GH, Mumbai, Maharashtra, India

Received: 26 December 2020

Accepted: 04 February 2021

\section{* Correspondence:}

Dr. Neha Khairnar,

E-mail: neha.khairnar@gmail.com

Copyright: (c) the author(s), publisher and licensee Medip Academy. This is an open-access article distributed under the terms of the Creative Commons Attribution Non-Commercial License, which permits unrestricted non-commercial use, distribution, and reproduction in any medium, provided the original work is properly cited.

\begin{abstract}
Chronic kidney disease is a heterogenous group of disorders resulting from anatomical and physiological alterations in the kidney. Pregnancy might accelerate the renal disease-causing progression of renal failure, development of preeclampsia, anemia. This increases the risk of adverse fetomaternal outcomes including prematurity, fetal growth restriction, fetal deaths and development of hypertension, nephrotic syndrome, renal failure in the mother. Chronic kidney disease affects approximately $3 \%$ of pregnant women. This study was carried out to assess the course of chronic kidney disease in pregnancy and the effect it has on the fetal outcome. A case of chronic kidney disease presenting to the OBGY emergency unit was studied. a detail history was taken and examination was done. Baseline antenatal investigations were carried out. Special tests including renal Doppler, ophthalmoscopic examination, ECG, were done to assess the renal function. Treatment was started for control of hypertension and further progression of the disease. Pregnancy has adverse outcome when associated with acute kidney injury. Maternal hypertension and proteinuria are the major predisposing factors. Prematurity is one of the commonest fetal complications apart from growth restriction. Chronic kidney disease in pregnancy requires a multidisciplinary approach involving experienced obstetricians, nephrologists, radiologists, intensivists and neonatologists. The etiology, degree of renal dysfunction, development of additional obstetric complications determines the prognosis. Supportive therapy in the early course of the disease and timely definitive management as per the etiology is found to improve the feto-maternal outcome.
\end{abstract}

Keywords: Hypertension, Creatinine, Prematurity, Dialysis, Multidisciplinary approach

\section{INTRODUCTION}

Chronic kidney disease (CKD) is a heterogeneous group of disorders which occur due to alterations in the structure and function of the kidney. It increases the risk of adverse feto-maternal outcomes. These risks increase if there is increase in the severity of the underlying renal dysfunction, proteinuria, and the coexistence of hypertension. Pregnancy might accelerate the renal disease causing progression of renal failure, development of preeclampsia, development of nephrotic syndrome, anemic syndrome, IUGR and fetal death.

Chronic kidney disease affects approximately $6 \%$ of women in the reproductive age in developed countries, and $3 \%$ of pregnant women. It has been seen that maternal, fetal and renal outcomes are bad if and when associated with advanced renal dysfunction, hypertension, proteinuria and poorly controlled underlying primary renal disease. ${ }^{1}$

\section{CASE REPORT}

Patient, a 23 year old female, second gravida with a previous spontaneous abortion, a known case of essential hypertension since 13 weeks of her pregnancy due to grade II to III medico-renal disease with anaemia and preeclampsia, had presented to our tertiary center at 28.6 weeks with a blood pressure of $160 / 100 \mathrm{mmHg}$ and proteinuria. She had no premonitory symptoms. After 
admission she was continued on oral anti-hypertensives labetelol $100 \mathrm{mg}$ twice a day and nifedepin $10 \mathrm{mg}$ twice a day which were later increased to maximum doses.

On admission, her haemoglobin was $9.2 \mathrm{~g} \%$, BUN 42 $\mathrm{mg} / \mathrm{dl}$, S.creatinine $2.2 \mathrm{mg} / \mathrm{dl}$, liver function tests and electrolytes were in normal range. Urine routine microscopy was suggestive of albuminuria. Renal doppler was normal. Rest of her antenatal profile was normal. her sonography showed a single live intrauterine fetus of 24 weeks, adequate liquor and estimated fetal weight of $1.1 \mathrm{~kg}$ ( suggestive of a four weeks lag). Fundoscopy showed no evidence of papilledema/ retinopathy. ECG and 2D echo were within normal limits.

Autoimmune workup done showed Antinuclear antibody positive. C3,C4 levels were normal and APLA, ACLA, Lupus anticoagulant, p- ANCA, c- ANCA were negative.

Patient was continued on anti-hypertensives and started on low dose aspirin. Steroid cover for the fetus was given.

At 30.3 weeks of gestation eleven days post admission, patient went into metabolic acidosis which was evident from tachypnoea and blood gas studies for which injection sodium bicarbonate was given for correction with monitoring of arterial blood gas values. She was also started on steroids and immunosuppressants that is Inj methylprednisolone and tablet Azathioprine by the nephrologist.

Twelfth day post admission, she developed premonitory symptoms along with a rise in blood pressure which was not controlled on full dose of two oral anti-hypertensive drugs requiring injectable labetolol and thus prophylactic levetiracetam was started. After counselling of relatives, decision for induction of labour was taken with consultation of the team involving nephrologist neonatologist, anaesthetist and intensivist. Four hours post induction with PG E1 gel, she was taken up for cesarean section in view of fetal distress under spinal anesthesia (with fluid restriction input equal to output, only one pint of intravenous fluid was given intraoperatively). She delivered a female child of $1.1 \mathrm{~kg}$ with Apgar score of $8 / 10$. Baby was admitted in the NICU.

Post operatively, vigilant monitoring of vitals and abdominal girth was done. IV antibiotics, steroids and analgesics were given with strict fluid restriction with input output monitoring. After starting orals 24hours post surgery, she was switched to oral drugs. Serum creatinine levels showed a rising trend for which she underwent 3 cycles of renal dialysis. On day 10 post operatively, suture removal was done as the wound was healthy. She was gradually tapered off Prednisolone and immunosuppressants were withheld. Anti-hypertensives were tapered.
She stayed in the ward till the baby was admitted in TCU. Baby was on RT feeds initially from the human milk bank and later started on mother's milk and gained weight eventually to $1.6 \mathrm{~kg}$. Patient was discharged on tablet Lasix 40 BD, tablet labetalol BD, tablet Prazopress 5 HS after 60 days of hospital stay.

\section{DISCUSSION}

In a case of $\mathrm{CKD}$, to get the best of pregnancy outcomes, we need to meticulously manage hypertension and proteinuria. Antenatally, measures should be taken to prevent the initiation of pre-eclampsia. Nephrotoxic and teratogenic medicines should be avoided. Renal dose of commonly used medications should be considered. Supportive treatment in early course of disease includes adequate hydration, correction of electrolyte imbalance and metabolic acidosis followed by definitive management according to the aetiology. End stage renal injury has to be treated with renal replacement therapy which is haemodialysis or renal transplant.

There is data to suggest that pregnancies have adverse outcomes when associated with acute kidney injury. Maternal hypertension and proteinuria are the major predisposing factors leading to negative outcomes. Hence, they should be pre-conceptionally counseled for the same. ${ }^{2}$

Whereas, with CKD, maternal hypertension and proteinuria not only deteriorate the CKD stage but also act as independent risk factors for poor outcome of pregnancy. Patients with immunologic diseases and glomerulonephritis are at a higher risk of developing a pre-eclampsia like picture with hypertension and proteinuria; often difficult to differentiate. ${ }^{3}$

Among the poor outcomes, prematurity is one of the commonest, as also seen with our patient. Prematurity brings about many short term and long tern risks for the babies like perinatal death, retinopathy, neurological problems; long terms risks for hypertension, cardiovascular complications. In our case, the baby had a NICU stay for two months but was discharged without any obvious complications. ${ }^{3}$

Pregnancy with CKD doesn't have a specific treatment as pregnancy in itself is not a disease as such. However, there are many drugs that are regularly used in the management of CKD, which cant be used with pregnancy. Hence the main line of management is proper monitoring, identification of potential complications ( hypertension, proteinuria, anaemia etc) and management of each. To assess the renal function during pregnancy is difficult due to presence of hyperfiltration and increased plasma volume. Creatinine clearance over $24 \mathrm{hrs}$ is the preferred assessment. $^{3}$

Whenever hypertension arises as a complication of pregnancy, it should be identified timely and treated. 
Over treatment should be avoided as it might compromise the utero placental circulation. Step wise treatment should be adapted. Drugs with the least side effects in pregnancy should be chosen. ${ }^{3}$

Hui et al in a study discussed the management of pregnancies in CKD patients in the context that pregnancy accelerates the disease progression. In 2015, Piccoli et al carried out a study stating the relation of pregnancy with increased loss of kidney function with each successive stage of CKD. In contrast to this, a recent study negated this association stating that renal dysfunction is not the only factor affecting the progression. Inadequately treated hypertension and proteinuria equally contribute to it. ${ }^{4}$

In a study by Jones et al, an important link between end stage kidney disease (ESKD) and hypertension in pregnancy was proved. These patients were likely to have hypertensive nephropathy later. ${ }^{5}$ In a classic study by Jones and Hayslett of 67 women with serum creatinine levels more than $1.4 \mathrm{mg} / \mathrm{dl}$ in pregnancy, $51 \%$ of women had no change in GFR as a result of pregnancy, but $31 \%$ had a decline in kidney function that persisted 6 months postpartum.

In another study by Ibarra-hernandez et al, Mexican women were seen to have a higher rate of adverse fetomaternal outcomes needing dialysis during and after the pregnancy. ${ }^{6}$

Hence, mode of delivery in women with chronic kidney disease should be based on usual obstetric indications, although more frequent prenatal assessments by an expert multidisciplinary team are desirable for the care of this particularly vulnerable patient population. Obstetricians in collaboration with nephrology team making combined management decisions is typically very helpful.

\section{CONCLUSION}

Pregnancy in women with chronic kidney disease is a complex therapeutic problem requiring a multidisciplinary approach involving experienced faculties from nephrology, anesthesia, obstetrics, intensive care unit, pathology, radiology, dialysis unit and neonatology. Determining the optimum time for termination of pregnancy depends on the condition of the mother, the condition of the fetus and the rate of progression of renal failure, and the pregnancy should be terminated is 35 weeks. The values of serum creatinine prior to pregnancy determines the prognosis. The other factors determining the prognosis are the degree of deterioration of renal function, development of additional obstetric complications and the specific etiological reasons that have leading to renal failure.

Funding: No funding sources

Conflict of interest: None declared

Ethical approval: Not required

\section{REFERENCES}

1. Webster P, Lightstone L, McKay DB, Josephson MA. Pregnancy in chronic kidney disease and kidney transplantation. Kidney International. Elsevier B.V. 2017;91:1047-56.

2. Koratala A, Bhattacharya D, Kazory A. Chronic Kidney Disease in Pregnancy. South Med J. 2017;110(9):578-85.

3. Cabiddu G, Castellino S, Gernone G, Santoro D, Moroni G, Giannattasio $\mathrm{M}$ et al. A best practice position statement on pregnancy in chronic kidney disease: the Italian Study Group on Kidney and Pregnancy. J Nephrol. Springer New York LLC. 2016;29:277-303

4. Hui D, Hladunewich MA. Chronic Kidney Disease and Pregnancy. Obstet Gynecol. 2019;133(6):118294.

5. Jones E, Rayner BL. Hypertension in pregnancy: A future risk for chronic kidney disease in South Africa. S Afr Med J . 2019;109(9):665-7.

6. Ibarra-Hernandez $\mathrm{M}$, Alcantar-Vallin $\mathrm{M}$ de la $\mathrm{L}$, Soto-Cruz A, Jimenez-Alvarado PM, Villa-Villagran F, Diaz-Avila J de J et al. Challenges in Managing Pregnancy in Underserved Women with Chronic Kidney Disease. Am J Nephrol . 2019;49(5):386-96.

Cite this article as: Khairnar N, Bora A, Bava AK. Impact of chronic kidney disease on fetomaternal outcome: a case report. Int J Reprod Contracept Obstet Gynecol 2021;10:1216-8. 